\title{
Incertidumbre de los índices de paisaje en el análisis de la estructura espacial
}

\author{
Uncertainty of landscape indices for spatial structure analysis
}

\author{
Adison Altamirano a*, Alejandro Miranda a , Cristian Jiménez ${ }^{a}$ \\ *Autor de correspondencia: ${ }^{\text {a }}$ Universidad de La Frontera, Departamento de Ciencias Forestales, Laboratorio de Análisis \\ Cuantitativo de Recursos Naturales, Casilla 54-D, Temuco, Chile, tel.: 56-45-325658, aaltamirano@ufro.cl
}

\begin{abstract}
SUMMARY
Landscape indices or metrics have been widely used to relate spatial patterns to ecological processes or ecological functions. Landscape indices have several uses and applications. However, there is evidence that landscape indices are very sensitive to remote sensing imagery analysis. The aim of this study was to assess the effect of accuracy of satellite imagery classification on landscape indices for the analysis of spatial structure. We used the supervised classification method with different classification strategies based on spectral and ancillary information. Eight images classified using different strategies were produced from a single satellite image. Landscape indices at landscape and class level were computed. Overall, results showed that images with higher classification accuracy have lower coefficient of variation of landscape indices. Some landscape indices as Shannon and Simpson diversity, percentage of like adjacencies, and aggregation index showed lower sensitivity to the increase of classification accuracy. However, other indices as patch density and core area were more sensitive. Sources of error in the satellite imagery analysis are very diverse. Hence, caution is advised in the analysis of spatial structure using landscape indices.
\end{abstract}

Key words: native forest, supervised classification, landscape metrics, remote sensing.

\section{RESUMEN}

Los índices o métricas de paisaje han sido ampliamente utilizados para relacionar patrones espaciales con procesos o funciones ecológicas. Si bien, su utilización y aplicaciones son amplias, se ha encontrado que los índices de paisaje son muy sensibles a algunos aspectos relacionados con el análisis de imágenes de sensores remotos. El objetivo de este estudio fue evaluar el efecto de la exactitud de clasificación de imágenes satelitales en los índices de paisaje para el análisis de la estructura espacial. Se utilizó el método de clasificación supervisada con distintas estrategias basadas en información espectral y auxiliar. A partir de una imagen satelital se generaron un total de ocho imágenes clasificadas con las distintas estrategias. Se calcularon índices de paisaje a nivel de paisaje y clase derivados de cada una de las imágenes clasificadas. Los resultados muestran que, en general, las imágenes con mayor exactitud presentan menores valores de coeficiente de variación en los índices de paisaje. Existen índices que muestran una menor sensibilidad frente al incremento en la exactitud de la clasificación, como los índices de diversidad de Shannon y Simpson, el porcentaje de adyacencias comunes y el índice de agregación. Sin embargo, otros índices muestran mayor sensibilidad, como la densidad de parches y el área núcleo. Las fuentes de error en el análisis de imágenes de sensores remotos son muy variadas. Debido a esto, se debe tener especial precaución en estos aspectos cuando se realice un análisis de la estructura espacial mediante índices de paisaje.

Palabras clave: bosque nativo, clasificación supervisada, métricas de paisaje, sensores remotos.

\section{INTRODUCCIÓN}

La ecología del paisaje ha estado estrechamente relacionada con el desarrollo de los sensores remotos (Groom et al. 2006). Sin embargo, los análisis de paisaje no siempre incorporan los nuevos métodos desarrollados por los especialistas en sensores remotos, lo cual ayudaría a incrementar la exactitud de los análisis (Newton et al. 2009). La mayor accesibilidad en las últimas décadas a datos de sensores remotos ha provocado gran interés en el análisis de la estructura y composición, además de la dinámica del paisaje, lo cual se ha realizado principalmente mediante algún tipo de categorización o clasificación de imágenes satelitales (Newton et al. 2009).
La clasificación de imágenes satelitales consiste básicamente en una categorización temática de la información obtenida por los sensores remotos. Información continua sobre la respuesta espectral de cada punto en el espacio geográfico (píxel) es llevada a distintas categorías o coberturas del suelo, las cuales pueden ser preestablecidas o no por el usuario (Lillesand et al. 2008). Esta aproximación permite cuantificar la dinámica espacial y temporal del paisaje en distintas escalas, teniendo una amplia gama de aplicaciones, tanto en ciencias ambientales como socioeconómicas.

Existe una gran cantidad de métodos que se han desarrollado para la generación de mapas temáticos mediante imágenes satelitales (Lu y Weng 2007). Sin embargo, el más ampliamente utilizado es el método de clasificación 
supervisada mediante un algoritmo de máxima verosimilitud. El mayor uso de este método se debe principalmente a su robustez, sencillez, rapidez en su aplicación, ya que se encuentra implementado en la mayoría de los software comerciales (Lu y Weng 2007). Una etapa fundamental dentro de la clasificación de imágenes satelitales es la evaluación de la exactitud de la clasificación, para lo cual se han desarrollado distintos métodos. El método más utilizado es la matriz de confusión (Lillesand et al. 2008), la cual entrega como resultado el porcentaje de puntos de control en terreno que está siendo correctamente clasificado en cada una de las categorías (Lillesand et al. 2008). En general, la evaluación de la clasificación de imágenes satelitales se mide en términos de la exactitud global de la clasificación, lo cual no representa la exactitud de las distintas categorías, si no una visión global de cómo se ha realizado el proceso de clasificación. En función del objeto de estudio y del paisaje estudiado, se utilizan categorías que agrupan distintas coberturas del suelo como agua, suelos desnudos, zonas urbanas, terrenos agrícolas, praderas y los distintos tipos de vegetación nativa o exótica presente en el área de estudio. Cada una de estas clases tiene distintos grados de dificultad en su identificación, siendo lo más sencillo identificar entre las clases de vegetación y cualquier otra clase o cobertura del suelo (cuerpos de agua, suelo desnudo, zonas urbanas, entre otros), y lo más complejo diferenciar entre las distintas clases de vegetación (Haapanem et al. 2004). Al ser la exactitud global un resumen de la exactitud de todas las clases, aquellas que presentan menor dificultad en su identificación y que tienen valores de exactitud cercanos al $100 \%$, pueden estar contribuyendo a la obtención de una exactitud global mayor, que no necesariamente represente la exactitud con la cual se está clasificando la o las coberturas de principal interés, como por ejemplo el bosque. Al ser las imágenes clasificadas uno de los principales insumos para los análisis de estructura espacial del paisaje, se producen variados efectos en función de la exactitud de la imagen clasificada. Esto se debe a que los errores que se producen en la categorización de las coberturas de interés genera una incertidumbre no cuantificada en los análisis posteriores mediante índices de paisaje.

Uno de los métodos más tradicionales para el análisis de la estructura espacial del paisaje es a través del cálculo de índices o métricas de paisaje. Estos índices han sido ampliamente utilizados para relacionar patrones espaciales con procesos o funciones ecológicas, recibiendo mucha atención de parte de la ecología del paisaje en las últimas décadas (Turner 2005). De acuerdo a McGarigal et al. (2002) los índices de paisaje corresponden a algoritmos que cuantifican una determinada característica espacial a nivel de parche, clase o paisaje, lo cual es realizado exclusivamente con mapas categorizados que provienen en su mayoría de la clasificación de imágenes de sensores remotos (Newton et al. 2009). Si bien, su utilización y aplicaciones son amplias, se ha encontrado que los índices de paisaje son muy sensibles a algunos aspectos relacionados con el análisis de imágenes de sensores remotos. Dentro de estos destacan la resolución espacial de las imágenes (Wickham y Riitters 1995, Baldwin et al. 2004), la extensión del área de estudio (Saura 2001, Baldwin et al. 2004), el método de clasificación (Mas et al. 2010), el número de clases utilizadas para la clasificación (Buyantuyev y Wu 2006), entre otros. Esto implica que las fuentes de incertidumbre en el análisis de la estructura espacial del paisaje, mediante índices de paisaje, pueden provenir de distintas fuentes en el proceso de análisis de imágenes satelitales, lo cual puede hacer muy variable los resultados obtenidos mediante este método.

La exactitud con la cual se logre representar la realidad mediante una clasificación puede tener una influencia difícil de cuantificar en el cálculo de índices de paisaje. Shao et al. (2001) informan un efecto de la exactitud de la clasificación en los índices de paisaje, lo cual se obtuvo a través de distintos métodos de clasificación de imágenes satelitales. En este estudio, los índices tienden, en general, a presentar una menor variación con mayor exactitud de clasificación, efecto que se muestra dependiente del índice analizado. Si bien Shao et al. (2001) señalan un efecto de la exactitud de clasificación, los métodos de clasificación fueron mezclados, utilizando clasificación supervisada, clasificación no supervisada, transformación mediante el análisis de componentes principales y transformación para destacar brillo, verdor y humedad (conocido como tasseled cap). Mas et al. (2010) reportan que una misma imagen clasificada con distintos métodos, a pesar de tener el mismo valor o muy similar de exactitud de clasificación, pueden producir distintas configuraciones espaciales del paisaje. Esto se explica porque distintos métodos o técnicas pueden generar la aparición o desaparición de pequeños parches, y asimismo, parches de mayor tamaño aparezcan conectados o desconectados (Mas et al. 2010). Debido a esto, la utilización de distintos métodos de clasificación podría tener un efecto en el análisis de la configuración espacial del paisaje incluso mayor que la exactitud de la clasificación.

El propósito de este trabajo es evaluar el efecto de la exactitud de la clasificación de imágenes satelitales en los índices de paisaje para el análisis de la estructura espacial. La exactitud de la clasificación de imágenes satelitales representa la confiabilidad con que se realizó la categorización del paisaje, por lo cual se espera que la mayor exactitud de la clasificación de las imágenes implique una mayor precisión en los índices tanto de paisaje como de clase. En este trabajo se utiliza el método de clasificación supervisada con distintas estrategias, la cuales son generadas a partir de distintos niveles de información espectral y auxiliar que permiten crear un gradiente de exactitud de clasificación.

\section{MÉTODOS}

Área de estudio. El área de estudio se encuentra ubicada en el suroeste de la región de Los Ríos, abarcando también el 
noroeste de la región de Los Lagos. Se extiende sobre una superficie de $3.720 \mathrm{~km}^{2}$ y elevaciones que varían aproximadamente entre 0 y $950 \mathrm{~m}$ s.n.m., entre $73^{\circ} 0^{\prime}$ y $73^{\circ} 50^{\prime} \mathrm{O}$, y $30^{\circ} 50^{\prime}$ a $40^{\circ} 25^{\prime} \mathrm{S}$ (figura 1). El área abarca parte de la Cordillera de la Costa y la Depresión Intermedia de las regiones antes mencionadas. La Cordillera de la Costa se encuentra en general cubierta por bosque nativo continuo compuesto mayormente por bosque laurifolio costero de Weinmannia trichosperma Cav. y Laureliopsis philippiana (Looser) Schodde y en menor proporción por bosque laurifolio templado de Nothofagus dombeyi (Mirb.) Oerst y Eucryphia cordifolia Cav., y bosque resinoso templado costero de Fitzroya cupressoides (Molina) Johnst. (Luebert y Pliscoff 2006). En la Depresión Intermedia predominan actividades económicas como la agricultura y la ganadería (CONAFCONAMA 2008), presentado bosques muy fragmentados de bosque caducifolio templado de Nothofagus obliqua (Mirb.) Oers. y Laurelia sempervirens (Ruiz et Pavón) Tul. (Luebert y Pliscoff 2006). El área muestra un paisaje heterogéneo con cuerpos de agua, zonas urbanas, áreas agrícolas y praderas, bosque nativo adulto y renovales, matorrales y plantaciones de especies exóticas, observándose un gradiente de intervención antrópica desde la depresión intermedia hacia la Cordillera de la Costa (CONAF-CONAMA 2008).

Datos. Se utilizó una imagen georreferenciada del sensor ASTER (Advanced Spaceborne Thermal Emission and Reflection Radiometer), desarrollado para obtener imágenes de alta resolución espacial y espectral. El sensor capta información en tres regiones espectrales diferentes: la región del visible e infrarrojo cercano (VNIR) compuesta por tres bandas espectrales con una resolución espacial de 15 m, la región del infrarrojo de onda corta (SWIR), con seis bandas espectrales de $30 \mathrm{~m}$ de resolución espacial, y la región del infrarrojo térmico (TIR) con cinco bandas espectrales de $90 \mathrm{~m}$ de resolución espacial. Para el presente estudio se utilizó una imagen adquirida el 7 de septiembre de 2009, considerando sólo las bandas de las regiones espectrales VNIR y SWIR.

Pre-procesamiento de imágenes satelitales. La imagen fue adquirida con un nivel de procesamiento L1B, a la cual se le han aplicado los coeficientes de corrección radiométrica y geométrica, además de tener corregistrados los datos de VNIR y SWIR. Los datos fueron convertidos a radiancia espectral, de acuerdo al procedimiento reportado por Smith (2004). Considerando el nivel de procesamiento de ASTER L1B, sólo se aplicó la corrección topográfica y atmosférica a la imagen. Para corregir topográficamente la imagen se utilizó la función de Minnaert (Mariotto y Gutschick 2010) y el modelo de elevación digital adquirido por el sensor ASTER, el cual tiene una resolución espacial de $30 \mathrm{~m}$. El cálculo de la constante de Minnaert se realizó utilizando la extensión del software ArcView "ArcView Grid and Theme regression” (Jenness 2006). El proceso de corrección topográfica se realizó utilizando el módulo "Topographic Normalize" implementado en el software ERDAS IMAGINE 2010. Para mayor detalle sobre el proceso de corrección topográfica ver Mariotto y Gutschick (2010). La corrección atmosférica se realizó mediante el método de substracción de píxeles oscuros, utilizando como objetos oscuros los cuerpos de agua presentes en la zona.

A las bandas espectrales de la región del SWIR se les aplicó un proceso de remuestreo automático (conocido como resample) para hacerlas compatibles con las bandas espectrales de la región del VNIR con una resolución espacial de

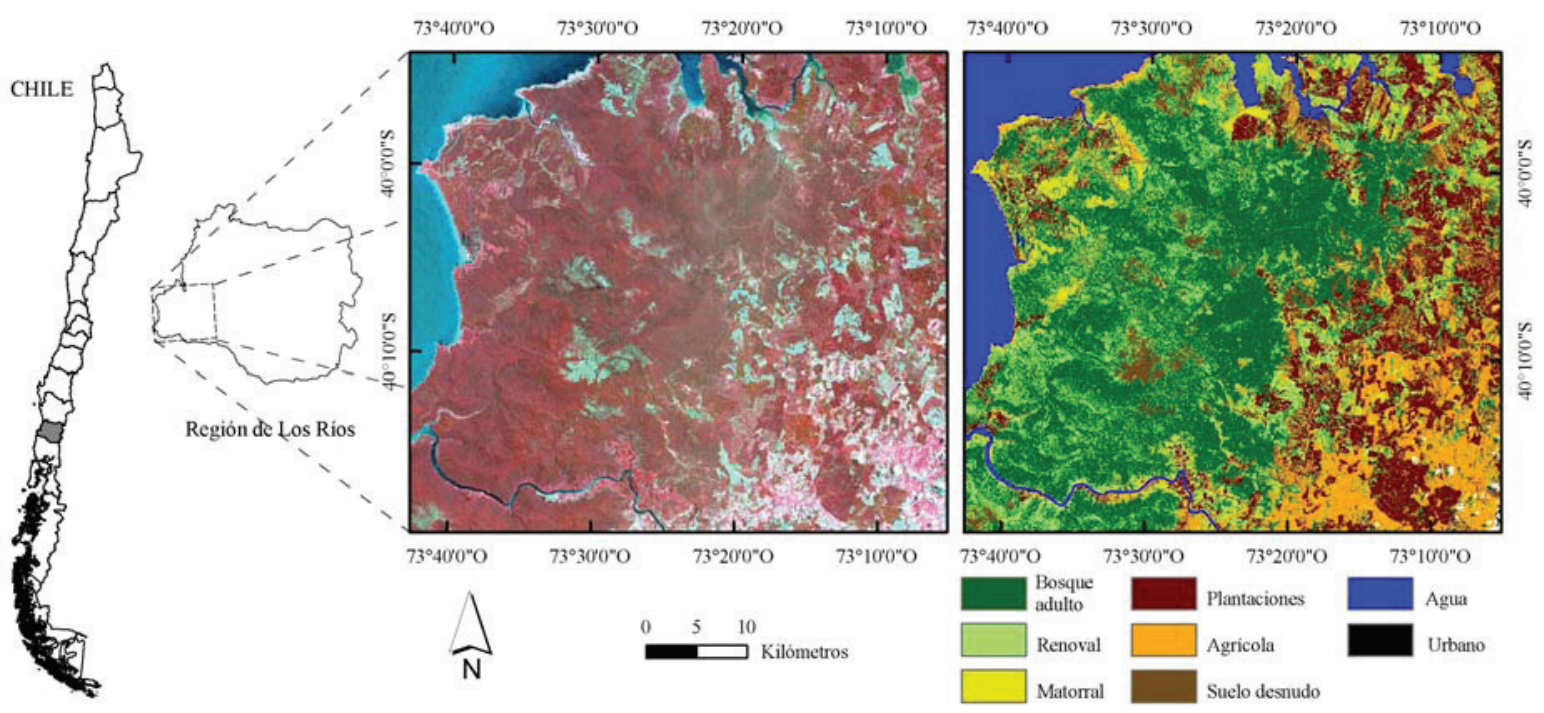

Figura 1. Imagen en composición de color infrarrojo y mapa de las principales coberturas del suelo del área de estudio. Infrared composite image and map of main land cover in the study area. 
15 m. Se dejó como grilla de trabajo las bandas del VNIR, obteniendo una imagen satelital con nueve bandas espectrales de $15 \mathrm{~m}$ de resolución espacial para la clasificación.

Clasificación supervisada. La clasificación temática de la imagen se realizó mediante el método de clasificación supervisada utilizando el algoritmo de máxima verosimilitud (Lillesand et al. 2008). Se hizo una campaña inicial de terreno para entrenar al clasificador en el reconocimiento de las distintas coberturas del suelo, y para establecer las primeras áreas de entrenamiento con la información de terreno. Además se utilizó información auxiliar, usando como referencia cartográfica las cubiertas digitales de CONAFCONAMA (2008). Para evitar posibles cambios en los criterios de clasificación, todo el proceso de clasificación fue realizado por la misma persona. La imagen se categorizó en ocho coberturas del suelo: bosque adulto, renoval, plantaciones (plantaciones forestales de especies exóticas, principalmente Pinus radiata D. Don y Eucalyptus sp.), matorral, terrenos agrícolas (incluye también praderas), agua, suelo desnudo y zonas urbanas (figura 1).

La clasificación supervisada se realizó usando distintos niveles de información, para observar los cambios en la exactitud de la clasificación de la vegetación y su efecto en los índices de paisaje. Se construyeron varias imágenes con un número distinto de bandas, utilizando la misma escena. La primera de ellas con las primeras tres bandas, la segunda con las primeras cuatro bandas, agregando así una banda espectral más hasta completar las nueve bandas espectrales que contienen la información de las regiones del VNIR y SWIR, generando un total de siete imágenes. Adicionalmente, se utilizó información auxiliar, lo cual consiste en agregar adicionalmente a las bandas espectrales (como una banda más) información que puede ser extraída de las mismas bandas espectrales a través de índices, cal- culados mediante algebra de bandas (Lillesand et al. 2008). Como información auxiliar fue utilizado el índice normalizado de diferencia infrarroja (NDII), el cual fue calculado como la diferencia entre la banda del infrarrojo cercano y la banda del infrarrojo medio, dividido por su suma. Este índice representa el stress hídrico de la vegetación (Bannari et al. 1995). De esta manera, se generaron un total de ocho imágenes clasificadas de la misma área de estudio con distintos niveles de información (cuadro 1). Cada imagen fue clasificada independientemente, utilizando las mismas áreas de entrenamiento para poder comparar los efectos de los distintos niveles de información en la exactitud de la clasificación, en donde la única diferencia entre cada clasificación es el nivel de información que contiene la imagen.

En una clasificación supervisada por píxel es común la aparición del efecto sal y pimienta (salt and pepper) (Lillesand et al. 2008), el cual se caracteriza por la presencia de coberturas homogéneas salpicadas por píxeles individuales pertenecientes a otras categorías. Para disminuir este efecto, a cada imagen clasificada se le realizó un filtro de moda de 3x3 píxeles, el cual cambia el valor central por el valor dominante dentro de la ventana definida, generalizando la imagen clasificada y eliminando los píxeles aislados (Mas et al. 2010).

Evaluación de la exactitud de la clasificación. La evaluación de la exactitud se realizó para las ocho imágenes clasificadas con las distintas estrategias, mediante la construcción de una matriz de confusión (Congalton y Green 1999). Se realizó un muestreo aleatorio estratificado de puntos de control en la imagen clasificada, con 50 puntos por cada cobertura del suelo utilizada en la clasificación (Lillesand et al. 2008, Chuvieco 2010). Se eliminaron los puntos que coincidieran en un mismo parche o que estuviesen ubicados en el borde de los mismos (Lillesand et al.

Cuadro 1. Estrategias de clasificación aplicadas mediante el método supervisado en el área de estudio. EGL = exactitud global, EBN = exactitud clase bosque nativo (unión de las coberturas bosque adulto y renoval), ERE = exactitud clase renoval, EBA = exactitud clase bosque adulto.

Classification strategies applied using the supervised method in the study area. EG=Overall accuracy, EBN=Accuracy of native forest class, $\mathrm{ERE}=$ Accuracy of second growth forest class, EBA= Accuracy of old growth forest class.

\begin{tabular}{llcccc}
\hline Estrategia & \multicolumn{1}{c}{ Descripción } & EGL (\%) & EBN (\%) & ERE (\%) & EBA (\%) \\
\hline B3 & banda 1 $(0,52-0,6 \mu \mathrm{m})+$ banda 2 $(0,63-0,69 \mu \mathrm{m})+$ & 74 & 54 & 46 & 62 \\
B4 & banda 3 $(0,78-0,86 \mu \mathrm{m})$ & 84 & 78 & 76 & 80 \\
B5 & B3 + banda 4 $(1,6-1,7 \mu \mathrm{m})$ & 86 & 79 & 86 & 72 \\
B6 & B4 + banda 5 $(2,145-2,185 \mu \mathrm{m})$ & 87 & 85 & 92 & 78 \\
B7 & B5 + banda 6 $(2,185-2.225 \mu \mathrm{m})$ & 87 & 83 & 90 & 76 \\
B8 & B6 + banda 7 $(2,235-2,285 \mu \mathrm{m})$ & 88 & 83 & 92 & 74 \\
B9 & B7 + banda 8 $(2,295-2,365 \mu \mathrm{m})$ & 88 & 87 & 92 & 82 \\
NDII & B8 + banda 9 $(2,36-2,43 \mu \mathrm{m})$ & 84 & 85 & 90 & 80 \\
\hline
\end{tabular}


2008). La asignación de la cobertura de suelo a cada punto de control, se realizó mediante una campaña de terreno y revisión de fotografías aéreas e imágenes satelitales de alta resolución espacial para zonas de difícil acceso. Se utilizaron los mismos puntos de control para la evaluación de la exactitud de todas las imágenes clasificadas con las distintas estrategias.

Cálculo de índices de paisaje. El cálculo de los índices de paisaje se realizó con el software FRAGSTATS 3.3 (McGarigal et al. 2002). Se calcularon índices de paisaje a nivel de paisaje, considerando el paisaje completo con todas las coberturas del suelo; y de clase, los cuales son calculados a cada una de las coberturas del suelo independientemente. Se seleccionaron índices de paisaje comúnmente utilizados en análisis de configuración espacial y fragmentación del paisaje (McGarigal et al. 2002).

A nivel de paisaje se calcularon: a) densidad de parches (DP), b) longitud total de borde (LB), c) porcentaje de adyacencias comunes (AC), d) índice de diversidad de Shannon (SH), y e) índice de diversidad de Simpson (SI). A nivel de clase se consideraron solo las coberturas de vegetación nativa, dado que la mayoría de los análisis de fragmentación se realizan en ecosistemas forestales (Newton et al. 2009). Para ello se utilizaron las coberturas de renoval y bosque adulto independientemente, $\mathrm{y}$ una de bosque nativo, resultante de la unión de las coberturas bosque adulto y renoval. A nivel de clase se calcularon cinco índices de paisaje: a) densidad de parches (DP), b) tamaño medio de parches (TM), c) área núcleo total (AN) (calcu- lado utilizando un borde de $100 \mathrm{~m}$ ), d) índice de proximidad media (IP) (calculado sobre un radio de búsqueda de 1000 m), y e) índice de agregación (IA). Una descripción detallada de cada índice de paisaje se puede encontrar en McGarigal et al. (2002). Para el cálculo de los índices se le eliminaron los parches de tamaño menor a 0,5 ha.

Análisis de sensibilidad de los índices de paisaje. Los mapas resultantes de la aplicación de las ocho estrategias de clasificación fueron divididos en tres grupos de acuerdo a su exactitud (cuadro 2). De esta manera, dos grupos contaron con tres estrategias de clasificación y un grupo con dos estrategias distintas, donde el grupo 1 agrupa las imágenes que obtuvieron la menor exactitud, el grupo 2 las imágenes con una exactitud media y el grupo 3 las imágenes que obtuvieron la mayor exactitud global o por clase. Para el análisis de los índices a nivel de paisaje, los mapas fueron agrupados de acuerdo a la exactitud global. En el caso de los índices a nivel de clase, éstos fueron agrupados de acuerdo a la media entre la exactitud del productor y la exactitud del usuario para cada clase.

Para evaluar la sensibilidad de los índices de paisaje a la exactitud de la clasificación se calculó la desviación estándar de los valores de cada índice en su grupo. Este estadígrafo fue estandarizado para poder comparar los distintos índices a través del cálculo del coeficiente de variación. Este coeficiente provee una medida de la cantidad relativa de variación de cada índice como la proporción de la desviación estándar respecto al promedio del índice (Mas et al. 2010).

Cuadro 2. Exactitud máxima, mínima y media de acuerdo a los distintos grupos e índices de paisaje. Los índices a nivel de paisaje fueron agrupados de acuerdo a la exactitud global, y los índices de paisaje a nivel de clase (bosque nativo, renoval y bosque adulto) de acuerdo a la media entre la exactitud del productor y la exactitud del usuario.

Maximum, minimum, and mean accuracy for groups and landscape indices. Landscape level indices were grouped according to overall accuracy. Class level indices (Native forest, Second growth forest and old growth forest) were grouped according to the average of producer's and user's accuracy.

\begin{tabular}{|c|c|c|c|c|}
\hline Índices de paisaje & Grupo & Exactitud máxima (\%) & Exactitud mínima (\%) & Exactitud media (\%) \\
\hline \multirow[t]{3}{*}{ Paisaje } & 1 & 84 & 74 & 80 \\
\hline & 2 & 87 & 86 & 87 \\
\hline & 3 & 88 & 88 & 88 \\
\hline \multirow[t]{3}{*}{ Bosque nativo } & 1 & 79 & 54 & 70 \\
\hline & 2 & 85 & 83 & 84 \\
\hline & 3 & 87 & 85 & 86 \\
\hline \multirow[t]{3}{*}{ Renoval } & 1 & 86 & 46 & 69 \\
\hline & 2 & 90 & 92 & 91 \\
\hline & 3 & 92 & 92 & 92 \\
\hline \multirow[t]{3}{*}{ Bosque adulto } & 1 & 74 & 62 & 69 \\
\hline & 2 & 80 & 76 & 78 \\
\hline & 3 & 82 & 80 & 81 \\
\hline
\end{tabular}




\section{RESULTADOS}

Exactitud de la clasificación. La exactitud global de la clasificación varió entre $74 \%$ y $88 \%$ (cuadro 1) para las ocho estrategias de clasificación aplicadas en el área de estudio (figura 2). La menor exactitud global se alcanzó utilizando las tres primeras bandas espectrales (B3), las cuales pertenecen a la región del visible e infrarrojo cercano (VNIR). Por otra parte, la mayor exactitud global se alcanzó utilizando las nueve bandas espectrales (B9). Los resultados observados para la exactitud de clasificación de las clases bosque nativo, renoval y bosque adulto muestran la misma tendencia. Sin embargo, se observa que la mayor y menor exactitud se alcanza en la clase renoval con $46 \%$ y $92 \%$. La exactitud de la clase bosque adulto varía entre $62 \%$ y $82 \%$. En tanto, la exactitud del bosque nativo (que incluye a las clases renoval y bosque adulto) varía entre $54 \%$ y $87 \%$. Con estos resultados se generaron tres grupos de imágenes en un gradiente de exactitud, donde el grupo 1 agrupa las imágenes que obtuvieron la menor exactitud, el grupo 2 las imágenes con una exactitud media y el grupo 3 las imágenes que obtuvieron la mayor exactitud global o por clase, obteniendo grupos distintos para los distintos niveles de análisis y para cada cobertura.

Variación de los índices de paisaje. Los índices a nivel de paisaje presentan valores máximos de coeficiente de variación de $20 \%$. Aquellos índices que presentaron un mayor coeficiente de variación fueron DP y LB (cuadro 3). Por el contario, se observaron variaciones menores en AC, SH y SI, ya que su cálculo no está relacionado con la forma y tamaño de los parches, si no más bien a la resolución temática de las imágenes, la cual es constante para las distintas estrategias.

A nivel de clase, para todas las coberturas analizadas se observa gran variación en el área núcleo (AN), ya que este índice es muy sensible al efecto de sal y pimienta ob-

Cuadro 3. Estadísticos descriptivos de los índices de paisaje a nivel de paisaje y clase utilizando las diferentes estrategias de clasificación. $\mathrm{MIN}=$ mínimo, $\mathrm{MAX}=$ máximo, $\mathrm{PRO}=$ promedio, $\mathrm{DE}=$ desviación estándar; $\mathrm{CV}=$ coeficiente de variación; s.u. $=$ sin unidad, $\mathrm{DP}=$ densidad de parches, $\mathrm{LB}=$ longitud total de borde, $\mathrm{AC}=$ porcentaje de adyacencias comunes, $\mathrm{SH}=$ índice de diversidad de $\mathrm{Shannon}, \mathrm{SI}=$ índice de diversidad de Simpson, $\mathrm{TM}$ = tamaño medio de parches, $\mathrm{AN}$ = área núcleo total, IP = índice de proximidad media, $\mathrm{IA}=$ índice de agregación.

Statistics of indices at landscape and class level using different classification strategies. MIN=Minimum, MAX $=$ Maximum, $P R O=M e a n$, $\mathrm{DE}=$ Standard deviation, $\mathrm{CV}=$ Coefficient of variation, $\mathrm{s} . \mathrm{u} .=$ Without unit, $\mathrm{DP}=$ Patch density, $\mathrm{LB}=$ Total length of edge, $\mathrm{AC}=$ Percentage of like adjacencies, $\mathrm{SH}=$ Shannon diversity index, $\mathrm{SI}=$ Simpson diversity index, $\mathrm{TM}=$ Mean patch size, $\mathrm{AN}=$ Core area, $\mathrm{IP}=$ Mean proximity index, $\mathrm{IA}=\mathrm{Aggregation}$ index .

\begin{tabular}{|c|c|c|c|c|c|}
\hline Índice & MIN & MAX & PRO & $\mathrm{DE}$ & CV (\%) \\
\hline \multicolumn{6}{|l|}{ Paisaje } \\
\hline DP (N/100 ha) & 83,8 & 141,9 & 100,9 & 20,0 & 20 \\
\hline LB (km) & 81.700 & 118.519 & 91.922 & 11.494 & 13 \\
\hline AC (\%) & 76,1 & 83,5 & 81,4 & 2,3 & 3 \\
\hline SH (s.u.) & 1,79 & 1,91 & 1,83 & 0,04 & 2 \\
\hline SI (s.u.) & 0,81 & 0,83 & 0,82 & 0,01 & 1 \\
\hline \multicolumn{6}{|l|}{ Bosque nativo } \\
\hline DP (N/100 ha) & 0,7 & 1,3 & 1,1 & 0,2 & 18 \\
\hline TM (ha) & 16,5 & 34,2 & 22,0 & 5,8 & 26 \\
\hline AN (ha) & 5,7 & 727,0 & 341,8 & 206,9 & 61 \\
\hline IP (s.u.) & 71.453 & 143.281 & 86.491 & 23.423 & 27 \\
\hline IA (\%) & 72,3 & 83,8 & 80,5 & 3,5 & 4 \\
\hline \multicolumn{6}{|l|}{ Renoval } \\
\hline DP (N/100 ha) & 2,4 & 3,1 & 2,6 & 0,3 & 10 \\
\hline TM (ha) & 2,1 & 4,1 & 3,4 & 0,7 & 19 \\
\hline AN (ha) & 0,3 & 97,2 & 40,5 & 35,0 & 86 \\
\hline IP (s.u.) & 45,8 & 549,9 & 334,2 & 71,7 & 52 \\
\hline IA (\%) & 60,9 & 75,2 & 71,7 & 4,8 & 7 \\
\hline \multicolumn{6}{|l|}{ Bosque adulto } \\
\hline DP (N/100 ha) & 1,6 & 2,8 & 2,3 & 0,4 & 16 \\
\hline TM (ha) & 8,3 & 16,8 & 11,3 & 2,7 & 24 \\
\hline AN (ha) & 4,2 & 339,7 & 177,2 & 113,1 & 64 \\
\hline IP (s.u.) & 25.638 & 72.188 & 50.063 & 17.045 & 34 \\
\hline IA (\%) & 71,5 & 78,5 & 76,2 & 2,1 & 3 \\
\hline
\end{tabular}



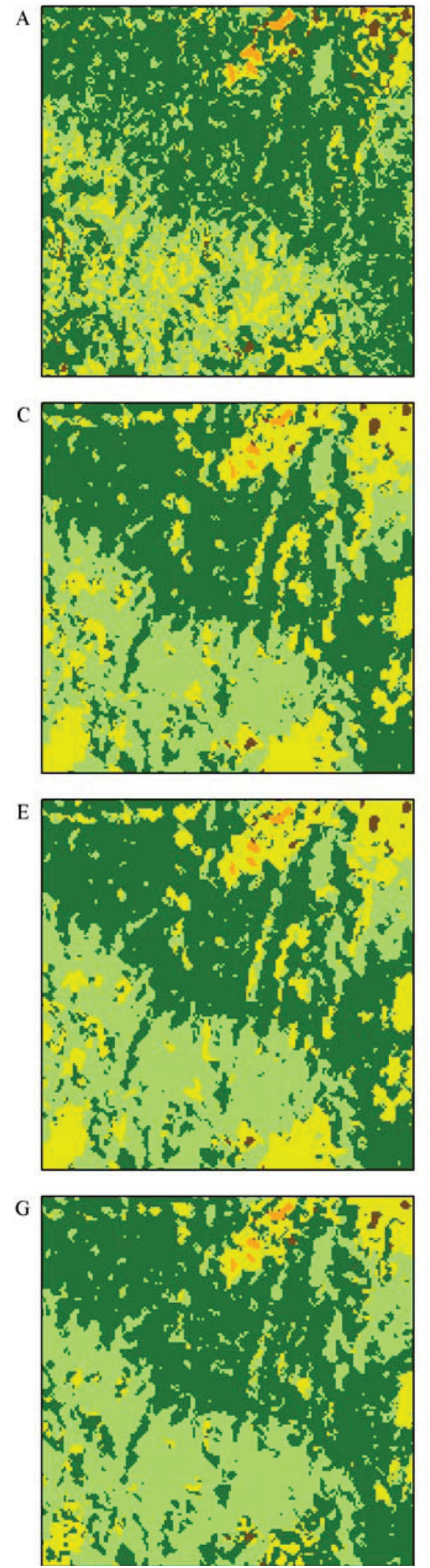
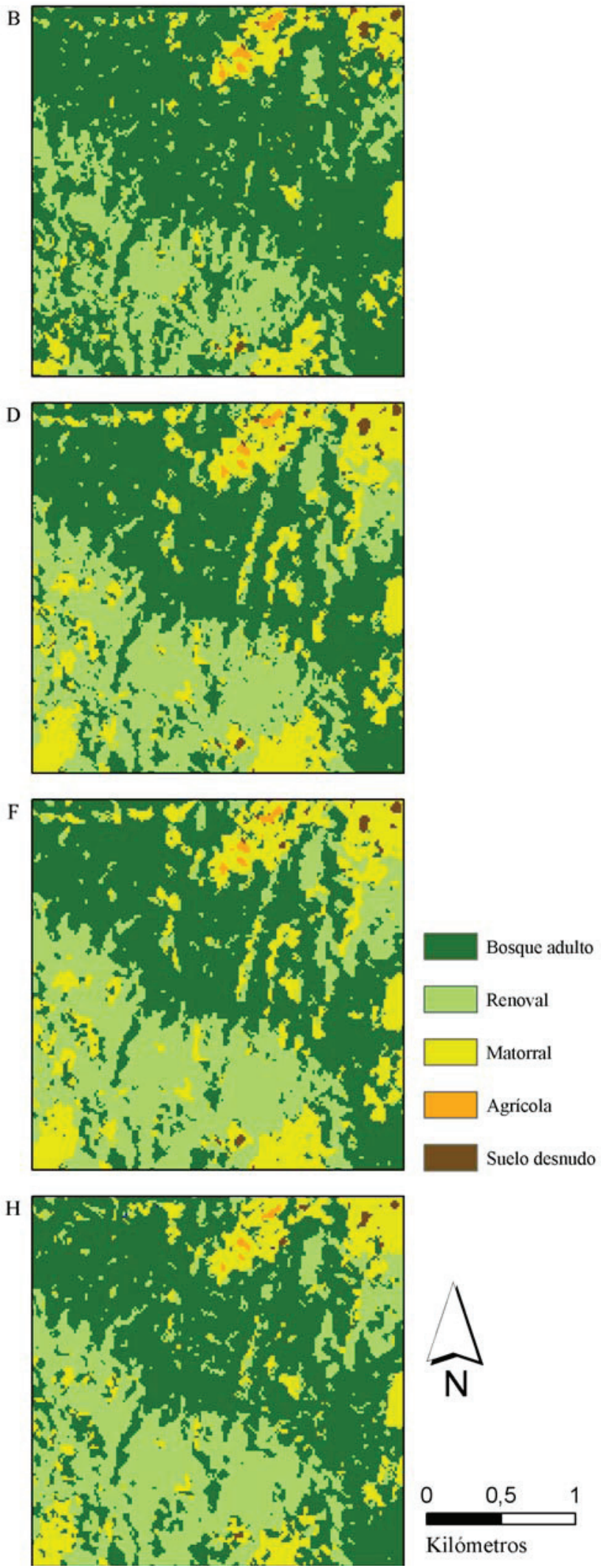

Figura 2. Mapa de cobertura del suelo con la aplicación del método de clasificación supervisada y las estrategias de clasificación utilizadas en parte del área de estudio: A) B3, B) B4, C) B5, D) B6, E) B7, F) B8, G) B9, y H) B9+NDII.

Map of land cover applying the supervised classification method and classification strategies in part of the study area: A) B3, B) B4, C) B5, D) B6, E) B7, F) B8, G) B9 H)B9+NDII. 
servado en la clasificación por píxel, debido a la aparición o desaparición de pequeños parches de otras coberturas al interior de las coberturas de vegetación (cuadro 3). Por otro lado, el índice de proximidad media (IP), presenta variaciones entre $27 \%$ y $52 \%$, lo cual no necesariamente se debe a la variación en el tamaño medio de los parches (TM), si no más bien a cambios en la densidad de parches (DP), modificando la distribución espacial de los fragmentos, y afectando la distancia entre ellos. Estas variaciones, cambian la configuración espacial de las distintas coberturas, la cual es muy sensible a la estrategia de clasificación.

Sensibilidad de los índices de paisaje a la exactitud de la clasificación. Los índices a nivel de clase presentan una mayor variación por grupo en comparación a los índices a nivel de paisaje. En particular, destaca el índice AN, el cual en todas las clases es el índice que presenta un mayor coeficiente de variación en cada uno de los grupos de exactitud de clasificación (figura 3 ).

A nivel de paisaje los valores del coeficiente variación en el grupo de mayor exactitud de clasificación (grupo 3) son inferiores a $5 \%$. El coeficiente de variación en este grupo de mayor exactitud es consistentemente menor en todos los índices en comparación con los otros dos grupos (figura 3A). De esta manera, los resultados muestran que, en general, una mayor exactitud de clasificación se asocia con una menor variación en los índices a nivel de paisaje. La mayor diferencia entre los grupos se presenta en los índices DP y LB, donde el coeficiente de variación de los grupos de menor exactitud (grupos 1 y 2) alcanza valores de $27 \%$ y $18 \%$, respectivamente. Una menor diferencia entre los distintos grupos se observa en los índices AC, SH y SI, con valores inferiores al $5 \%$ en el coeficiente de variación, considerando que corresponden a índices descriptivos principalmente de la composición del paisaje, lo que los hace más estables al cambio en estrategia de clasificación.

A nivel de clase, en el bosque nativo, los resultados muestran que los grupos de mediana y mayor exactitud de clasificación (grupos 2 y 3 ) tienen un menor valor de coeficiente de variación en los índices de paisaje en comparación al grupo de menor exactitud (grupo 1) (figura 3B), alcanzando las mayores diferencias en el índice de área núcleo (85 \%) en el grupo de menor exactitud.

En la clase renoval, el coeficiente de variación en el grupo de mayor exactitud de clasificación (grupo 3) muestra una tendencia más constante respecto a los demás (figura 3C). El grupo que presenta valores mayores de coeficiente de variación en todos los índices corresponde al de menor exactitud de clasificación (grupo 1). En este grupo el coeficiente de variación supera el $100 \%$ en los índices IP y AN, donde se producen las mayores diferencias con los otros dos grupos.
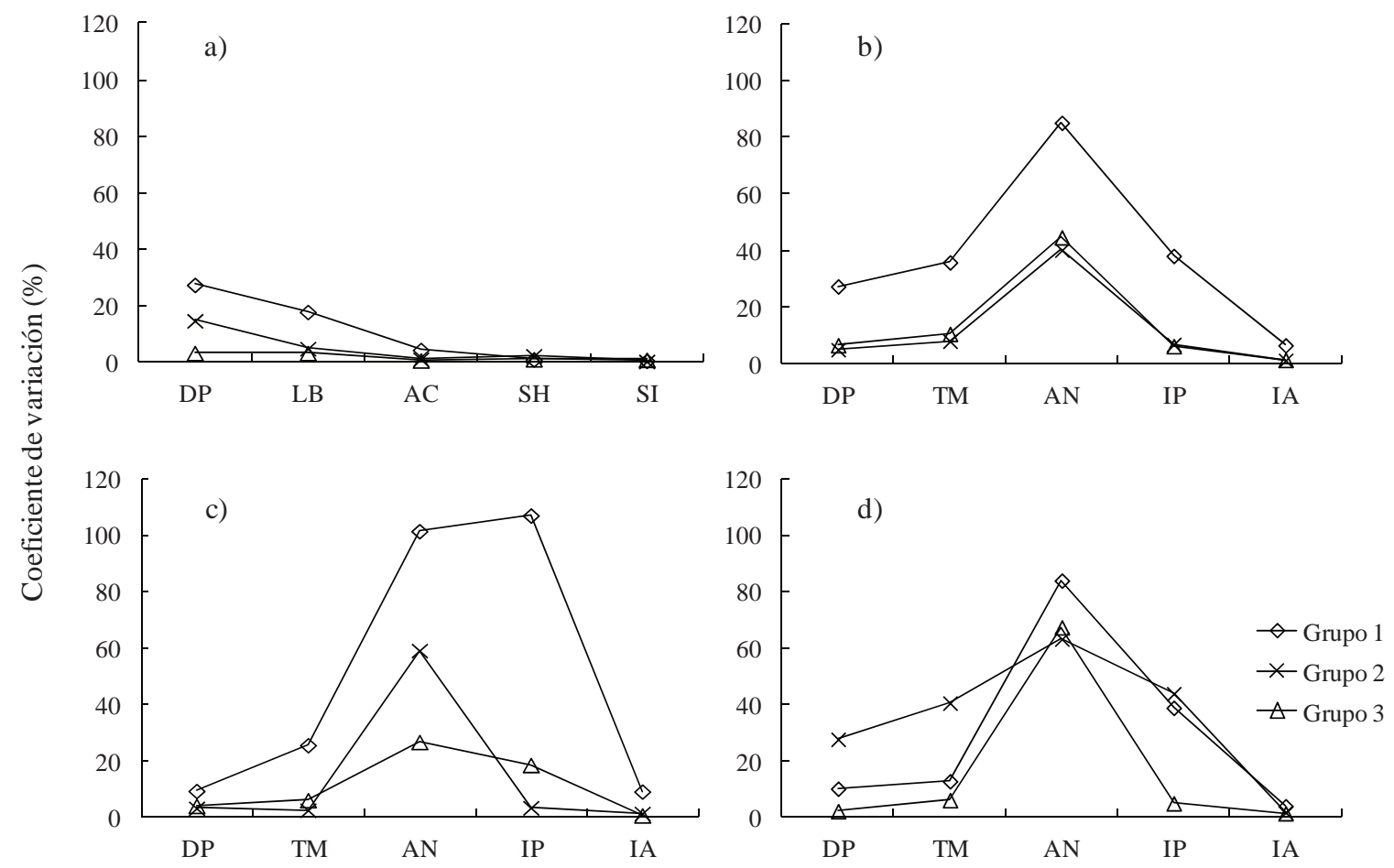

Figura 3. Coeficiente de variación (\%) de acuerdo a grupos de exactitud e índices a nivel de A) paisaje, y clase, B) bosque nativo, C) renoval y D) bosque adulto.

Coefficient of variation (\%) for accuracy groups and indices at A) landscape level, and class level B) native forest, C) second growth forest, and $\mathrm{D}$ ) old growth forest. $\mathrm{DP}=$ Patch density, $\mathrm{LB}=$ Total length of edge, $\mathrm{AC}=$ Percentage of like adjacencies, $\mathrm{SH}=$ Shannon diversity index, $\mathrm{SI}=$ Simpson diversity index, $\mathrm{TM}=$ Mean patch size, $\mathrm{AN}=$ Core area, $\mathrm{IP}=$ Mean proximity index, $\mathrm{IA}=$ Aggregation index . 
En la clase bosque adulto, los valores más bajos del coeficiente de variación se observan en el grupo de mayor exactitud (grupo 3), a excepción del índice AN, cuyo valor es de $67 \%$ (figura 3D). Todos los demás índices tienen valores inferiores a $6 \%$. Sólo en el índice AN, el coeficiente de variación es menor en el grupo de mediana exactitud (grupo 2). En esta clase el coeficiente de variación alcanza sus máximos valores en los grupos de menor (grupo 1) y mediana (grupo 2) exactitud de clasificación.

Los resultados reflejan en general una mayor sensibilidad a la exactitud de la clasificación en índices que describen las características estructurales de los fragmentos, en comparación con índices que tienen relación con la composición del paisaje.

\section{DISCUSIÓN Y CONCLUSIONES}

Los resultados respecto a la generación de un gradiente de exactitud de clasificación (cuadro 1) son consistentes con otros estudios que reportan este tipo de medidas de confiabilidad de los datos satelitales (Congalton y Green 1999, Lillesand et al. 2008). En este estudio sólo se utilizó información espectral, alcanzando un máximo de exactitud global de $88 \%$. Sin duda, el uso de otros métodos podría mejorar la exactitud global de la clasificación ( $\mathrm{Lu} \mathrm{y}$ Weng 2007). Sin embargo, el método utilizado fue seleccionado ya que representa el más ampliamente utilizado para la generación de mapas temáticos (Lu y Weng 2007, Lillesand et al. 2008, Newton et al. 2009).

Es común que el reporte de la incertidumbre de la clasificación sólo se restrinja a la exactitud global, la cual no representa la exactitud de las distintas clases de cobertura del suelo. En la mayoría de los estudios que aplican clasificación de imagen satelitales, la exactitud global está fuertemente influenciada por algunas coberturas del suelo que son altamente diferenciables espectralmente (ejemplo: cuerpos de agua, nieve, terrenos sin vegetación). Esto redunda en una mayor exactitud de este tipo de coberturas y, por ende, tiende a incrementar la exactitud global, lo cual no implica un incremento en la exactitud de las clases de interés. Este aspecto es muy relevante en estudios donde alguna de las coberturas del suelo identificadas es el objeto de estudio. Ejemplos de esto lo constituyen estudios de pérdida de bosque, fragmentación y degradación forestal, donde resulta complejo diferenciar las clases asociadas a la vegetación debido a su alta similitud espectral (Haapanen et al. 2004).

Una alternativa simple para mejorar la exactitud de la clasificación es el uso de información auxiliar respecto al contexto de los píxeles vecinos. Un ejemplo de esto es el uso de análisis de textura, lo cual puede ser útil para diferenciar entre coberturas de plantaciones forestales y bosque nativo, debido a que este último presenta una estructura horizontal más heterogénea que las plantaciones forestales. Este tipo de análisis permite un mayor grado de diferenciación entre algunas coberturas, tal como lo comprobaron Altamirano y Lara (2010) en bosques templados. Los análisis de este estudio no incluyen este tipo de información, a pesar de que se puede mejorar la exactitud disminuyendo el efecto sal y pimienta observado en la clasificación por píxel (Lillesand et al. 2008). La razón se debe a que esto produce una fuerte influencia en algunos índices como el número y el tamaño medio de los parches, mostrando posiblemente un paisaje menos fragmentado (Langford et al. 2006).

En términos generales, a nivel de paisaje y de clase, el grupo de imágenes analizadas con mayor exactitud muestran menores valores de coeficiente de variación en los índices de paisaje, revelando una mayor estabilidad de estos a medida que se incrementa la exactitud de la clasificación (figura 3). Esto es concordante con lo reportado por Shao et al. (2001), quienes relacionaron la variación de los índices de paisaje y la exactitud de clasificación. Sin embargo, esta tendencia no siempre se manifiesta, y en el presente estudio podría estar influenciada por el bajo número de muestras que conforman los grupos. Alcanzar máximos de exactitud de clasificación no garantiza reducir la variación en todos los índices de paisaje. Shao et al. (2001), en un análisis de 23 mapas con poca variación en la exactitud de clasificación, señalan que hubo una gran variación en los índices de paisaje calculados. Asimismo, Mas et al. (2010) reportan una diferencia significativa entre índices de paisaje calculados en imágenes clasificadas y el mismo valor de exactitud, pero utilizando métodos diferentes.

Los resultados revelan la existencia de índices que muestran una menor sensibilidad, y por tanto una mayor estabilidad frente al incremento en la exactitud de la clasificación (figura 3). Los análisis realizados no permiten distinguir si los índices a nivel de paisaje tienen una menor sensibilidad que los índices a nivel de clase como señala Mas et al. (2010). Esto se debe a que el único índice en común es DP, el cual no muestra una diferencia sustancial, observando mayores diferencias entre las distintas coberturas (figura 3). A nivel de paisaje, SH y SI corresponden a índices de diversidad, los cuales describen la composición del paisaje. Estos índices no consideran la configuración espacial del paisaje, sino la abundancia de los distintos tipos de parches en el paisaje (McGarigal et al. 2002). Por lo tanto, esto indicaría que las estrategias utilizadas afectan la configuración espacial del paisaje, pero no tendrían mayor influencia en su composición. Esta baja influencia en los índices de diversidad también se explica por la baja resolución temática elegida, presentando un bajo número de clases o coberturas del suelo. Otro índice que no se vio afectado $(\mathrm{CV}=3 \%)$ por las distintas estrategias utilizadas para clasificar es el índice AC (porcentaje de adyacencias comunes), siendo muy estable ante la exactitud de la clasificación. Esto podría indicar que la aplicación de las distintas estrategias, a pesar de modificar el área y el perímetro de los parches, no tuvo mayor influencia en la agregación de los píxeles a nivel de paisaje, siendo probablemente más sensible al método de clasificación. Lo anterior tam- 
bién indica que este índice a escala de paisaje sólo daría cuenta de cambios muy fuertes en el paisaje, no siendo el más adecuado para detectar cambios temporales en paisajes poco dinámicos. A nivel de clase, el índice IA muestra una baja variación (figura 3B, C y D), ya que su cálculo es indiferente del área de los parches, mostrando poca sensibilidad. El mismo comportamiento se presenta en AC a nivel de paisaje (figura 3A), donde el cambio en el tamaño y forma de los parches no tiene mayor influencia. Esto sugiere que la mayor sensibilidad se observa en índices que cuantifican atributos individuales de cada cobertura (DP, LB, TM, AN, IP), los cuales presentan una mayor sensibilidad en función de la estrategia o exactitud con la cual se generen los mapas temáticos, que aquellos índices que describen de manera más general el paisaje (SH, SI, IA). Esto puede tener una explicación en la dificultad específica de clasificar las distintas coberturas del suelo, siendo más sencillo cuantificar las coberturas sin vegetación, lo cual al hacer análisis generales de paisaje se ven aminorados por aquellas coberturas que presenta menores dificultades en su detección.

Las imágenes satelitales se han convertido en uno de los insumos más importantes en estudios de ecología del paisaje para evaluar dinámica de configuración espacial y fragmentación del hábitat utilizando índices de paisaje (Echeverría et al. 2006, Altamirano et al. 2007). Sin embargo, muchos estudios de este tipo omiten información respecto al procesamiento de las imágenes satelitales (por ejemplo, exactitud de clasificación) (Newton et al. 2009), lo cual tiene importantes implicancias en los análisis e interpretaciones posteriores (Shao et al. 2001). Algunos de los índices más utilizados en este tipo de estudios son la densidad de parches y el área núcleo, calculados a nivel de clase. Como ejemplo, en el estudio de Echeverría et al. (2006) estos índices muestran una variación temporal de $46 \%$ y $96 \%$, respectivamente, en un período de 25 años. En el presente estudio, el coeficiente de variación de estos índices fue de hasta $18 \%$ y $86 \%$, respectivamente (cuadro 3 ). Dados estos resultados, gran parte de la variación de los índices de paisaje en estudios multitemporales podría deberse al efecto de la exactitud de la clasificación y no a la dinámica del paisaje.

Una primera medida básica que contribuya a mejorar la fiabilidad de los índices de paisaje es utilizar técnicas que permitan alcanzar una mayor exactitud de clasificación. Shao et al. (2001) señalan que aunque la relación entre exactitud de clasificación e índices de paisaje no es lineal, cuando la exactitud de la clasificación se acerca al $90 \%$, los valores de los índices de paisaje tienden a estabilizarse. Los resultados indican que hay ciertos índices de paisaje que muestran ser menos sensibles a la exactitud de la clasificación (figura 3A), en particular, los índices que cuantifican la composición del paisaje. Por lo tanto, ante a una mayor incertidumbre con el análisis de datos satelitales sería preferible usar este tipo de índices (Mas et al. 2010). Uno de los problemas de los índices de pai- saje es que su distribución es desconocida, lo cual limita su comparación estadística (Turner et al. 2005). Para sobrellevar este problema una alternativa es la generación de intervalos de confianza para cada índice a través de la simulación de paisajes a partir de un paisaje neutral, con lo cual se puede obtener la distribución de los valores de un índice (Fortin et al. 2003). La incertidumbre asociada con el análisis de datos provenientes de imágenes satelitales es algo difícilmente evitable, sabiendo que no existe clasificación sin errores. Además, las fuentes de error pueden ser muy variadas (resolución de las imágenes, fecha de adquisición, áreas de comparación, métodos utilizados, experiencia del usuario, entre otras), debiendo tener precaución en estos aspectos cuando se realice un análisis de la estructura espacial mediante índices de paisaje.

\section{AGRADECIMIENTOS}

Este estudio fue financiado con el apoyo del proyecto FONDEF D08I1056 y la Dirección de Investigación de la Universidad de La Frontera. Queremos agradecer a los profesores Mauricio Reyes y Patricio Acevedo de la Universidad de La Frontera por facilitar la imagen satelital utilizada en el estudio.

\section{REFERENCIAS}

Altamirano A, A Lara. 2010. Deforestación en ecosistemas templados de la precordillera andina del centro-sur de Chile. Bosque 31(1): 53-64.

Altamirano A, C Echeverría, A Lara. 2007. Efecto de la fragmentación forestal sobre la estructura vegetacional de las poblaciones amenazadas de Legrandia concinna (Myrtaceae) del centro-sur de Chile. Revista Chilena de Historia Natural 80 (1): 27-42.

Baldwin DJB, K Weaver, F Schnekenburger, AH Perera. 2004. Sensitivity of landscape pattern indices to input data characteristics on real landscapes: implications for their use in natural disturbance emulation. Landscape Ecology 19: 255-271.

Bannari A, D Morin, F Bonn, AR Huete. 1995. A review of vegetation indices. Remote Sensing Reviews 13: 95-120.

Buyantuyev A, J Wu. 2006. Effects of thematic resolution on landscape pattern analysis. Landscape Ecology 22(1): 7-13.

CONAF-CONAMA (Corporación Nacional Forestal, CL - Comisión Nacional del Medioambiente, CL). 2008. Catastro de uso de suelo y vegetación. Monitoreo y actualización Región de Los Ríos, periodo 1998-2006. 19 p.

Congalton RG, K Green. 1999. Assessing the accuracy of remotely sensed data: principles and practices. Boca Raton, USA. Lewis Publishers. 137 p.

Echeverría C, D Coomes, J Salas, JM Rey-Benayas, A Lara, A Newton. 2006. Rapid deforestation and fragmentation of chilean temperate forests. Biological Conservation 130: 481-494.

Fortin MJ, B Boots, F Csillag, TK Remmel. 2003. On the role of spatial stochastic models in understanding landscape indices. Oikos 102: 203-12. 
Groom G, CA Mücher, M Ihse, T Wrbka. 2006: Remote sensing in landscape ecology: experiences and perspectives in a European context. Landscape Ecology 21: 391-408.

Haapanen R, AR Ek, ME Bauer, AO Finley. 2004. Delineation of forest/nonforest land use classes using nearest neighbor method. Remote Sensing of Environment 89: 265-271.

Jenness J. 2006. Grid and Theme Regression 3.1e (grid_regression.avx) extension for ArcView 3.x. Jenness Enterprises: Flagstaff, AZ, USA. Consultado 16 mar. 2011. Disponible en: http://www.jennessent.com/arcview/regression.htm

Langford W, SE Gergel, TG Dietterich, W Cohen. 2006. Map misclassification can cause large errors in landscape pattern indices: examples from habitat fragmentation. Ecosystems 9: 474-488.

Lillesand T, R Kiefer, J Chipman. 2008. Remote sensing and image interpretation. New York, USA. John Wiley, Sixth edition. 756 p.

Lu D, Q Weng. 2007. A survey of image classification methods and techniques for improving classification performance. International Journal of Remote Sensing 28(5): 823-870.

Luebert F, P Pliscoff. 2006. Sinopsis bioclimática y vegetacional de Chile. Santiago, Chile. Editorial Universitaria. 316 p.

Mariotto I, V P Gutschick. 2010. Non-Lambertian Corrected Albedo and Vegetation Index for Estimating Land Evapotranspiration in a Heterogeneous Semi-Arid Landscape. Remote Sensing 2(4): 926-938.

Mas JF, Y Gao, JAN Pacheco. 2010. Sensitivity of landscape pattern metrics to classification approaches. Forest Ecology and Management 259(7): 1215-1224.

McGarigal K, SA Cushman, MC Neel, E Ene. 2002. FRAGSTATS: Spatial Pattern Analysis Program for Categorical Maps. University of Massachusetts, Amherst, computer software program. Consultado 30 abr. 2011. Disponible en: http// www.umass.edu/landeco/research/fragstats/fragstats.html.

Newton A, R Hill, C Echeverria, D Golicher, JM Rey-Benayas, L Cayuela, S Hinsley. 2009. Remote sensing and the future of landscape ecology. Progress in Physical Geography 33: 528-546.

Saura S, J Martínez-Millán. 2001. Sensitivity of landscape pattern metrics to map spatial extent. Photogrammetric Engineering and Remote Sensing 67(9): 1027-1036.

Shao G, D Liu, G Zhao. 2001. Relationships of image classification accuracy and variation of landscape statistics. Canadian Journal of Remote Sensing 27:33-43

Smith AMS. 2004. How to Convert ASTER Radiance Values to Reflectance. College of Natural Resources, University Idaho. Consultado 20 nov. 2010. Disponible en: http//www. cnrhome.uidaho.edu/default.aspx?pid=85984

Turner MG. 2005. Landscape ecology: what is the state of the science? Annual Review of Ecology, Evolution and Systematics 36: 319-44.

Wickham JD, KH Riitters. 1995. Sensitivity of landscape metrics to pixel size. International Journal of Remote Sensing 16: 3585-94.

Recibido: 13.01 .12

Aceptado: 29.05.12 
